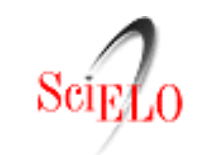

「artículos $\longrightarrow$ búsqueda de artículos

sumario anterior próximo autor materia búsqueda home alfab

Revista Científica

versión impresa ISSN 0798-2259

Rev. Cient. (Maracaibo) v.17 n.4 Maracaibo ago. 2007

\section{Constituyentes minerales del Morocoto Piaractus Brachypomus en el Orinoco Medio de Venezuela.}

\author{
Ángel González ${ }^{1 *}$, Arístide Márquez ${ }^{2}$, William Senior ${ }^{2}$ y Gregorio \\ Martínez 2
}

$1^{*}$ Instituto Limnológico, Universidad de Oriente, Caicara del Orinoco, estado Bolívar. TIf. (0284)6667474(0284)6667090. Cel. 04162477940. Email: angelgonzalez78@hotmail.com

2 Instituto Oceanográfico de Venezuela, UDO, Departamento de Oceanografía, Cumaná, estado Sucre.

\section{RESUMEN}

Se realizó un análisis de la concentración de algunos minerales como potasio, sodio, calcio, magnesio y hierro en el tejido muscular del morocoto Piaractus brachypomus del Orinoco medio en Venezuela, capturado en algunas lagunas de inundación con redes de enmalle. La concentración de estos minerales fue analizada mediante Absorción Atómica con Ilama de aire-acetileno y corrección de fondo de deuterio, utilizando un equipo Perkin Elmer 3100 acoplado con un automuestreador Perkin-Elmer AS-51. Se determinó un promedio 45,32 $\pm 0,52 \mu \mathrm{g} / \mathrm{g}$ en la concentración de hierro, $3497,48 \pm 107,06 \mu \mathrm{g} / \mathrm{g}$ en la concentración de calcio, $1528,95 \pm 29,25 \mu \mathrm{g} / \mathrm{g}$ en la concentración de magnesio, 3529,66 $\pm 65,24 \mu \mathrm{g} / \mathrm{g}$ en la concentración de sodio y 16488,90 $\pm 264,69 \mu \mathrm{g} / \mathrm{g}$ en la concentración de potasio. Un análisis de varianza (ANOVA) y prueba posteriori de Duncan, demostró que estas concentraciones presentaron diferencias significativas, con una mayor concentración de potasio, seguido del sodio y el calcio, y luego el magnesio y el hierro. Entre estos minerales las concentraciones de calcio y potasio presentaron correlación estadísticamente significativa. Los resultados constituyen un aporte al conocimiento de la composición química de $P$. brachypomus, uno de los peces de agua dulce más importante en el país desde el punto de vista comercial y en la acuacultura.

\section{Servicios Personalizados}

Articulo

Articulo en XML

Referencias del artículo

Como citar este

Traducción automática

Enviar articulo por email

Indicadores

Accesos

Links relacionados

Compartir

Otros $m$

Otros

Permalink
Citado por SciELO 
Palabras clave: Composición química de peces, constituyentes minerales en peces, peces de agua dulce, Piaractus brachypomus, río Orinoco.

9 Citavi ha encontrado 1 títulos que se pueden importar directamente. Haga clic aquí para importar los títulos.

\section{ABSTRACT}

An analysis of the concentrations of some mineral as potassium, sodium, calcium, magnesium and iron was made in the muscular tissue of the morocoto Piaractus brachypomus from the middle Orinoco River in Venezuela, captured in some flood lagoons with gillnet. The concentration of these minerals was analyzed by Atomic Absorption with flame of air-acetylene and correction of deuterium, using a team Perkin Elmer 3100 coupled with an autosampler Perkin-Elmer ACE-51. An average of $45.32 \pm 0.52 \mu \mathrm{g} / \mathrm{g}$ was determined in the iron concentration, $3497.48 \pm 107.06 \mu \mathrm{g} / \mathrm{g}$ in the concentration of calcium, $1528.95 \pm 29.25 \mu \mathrm{g} / \mathrm{g}$ in the concentration of magnesium, $3529.66 \pm 65.24 \mu \mathrm{g} / \mathrm{g}$ in the concentration of sodium and $16488.90 \pm 264.69 \mu \mathrm{g} / \mathrm{g}$ in the concentration of potassium. A variance analysis (ANOVA) and Duncan test later, demonstrated that these concentrations presented significant differences, with a bigger concentration of potassium, followed by the sodium and the calcium, and then the magnesium and the iron. Among these minerals the concentrations of calcium and potassium presented correlation statistically significant. The results constitute a contribution to the knowledge of the chemical composition of $P$. brachypomus, one of the freshwater fish most important in the country from the commercial point of view and in the aquaculture.

Key words: Fish chemical composition, fish mineral constituents, freshwater fish, Piaractus brachypomus, Orinoco River.

Recibido: 27 / 06 / 2006. Aceptado: 19 / 01 / 2007.

\section{INTRODUCCIÓN}

Piaractus brachypomus, conocida comúnmente en Venezuela como morocoto y pirapitanga en el Amazonas, es una especie de agua dulce perteneciente a la familia Characidae, subfamilia Characiforme, de la clase Actinopterygii. Se encuentra ampliamente distribuida en Suramérica, en las cuencas del Amazonas y el Orinoco (16), incluyendo a los Ilanos venezolanos (17) y la planicie de inundación del Orinoco (20). La exquisitez de su carne la convierte en una de las especies de agua dulce más solicitada para el consumo, adquiriendo mucha importancia desde el punto de vista pesquero $(20,21)$. Desde este punto de vista, en los desembarques fluviales de Venezuela, la producción de $P$. brachypomus se ha mantenido durante los últimos años en alrededor de las 1000 toneladas anuales, con un $2 \%$ de los desembarques totales aproximadamente; procediendo casi la totalidad de los desembarques del río Orinoco, especialmente del delta y del Orinoco medio (22). Desde el punto de vista de la acuacultura, ha sido exitosamente utilizada como especie de cultivo al igual que el híbrido obtenido de su cruce con la cachama, Colossoma macropomum, conocido como cachamoto (22). Como un aporte al conocimiento de la composición química de $P$. brachypomus, en este trabajo se determinó su concentración en algunos minerales como el potasio, sodio, calcio, magnesio y hierro, considerados importantes en el metabolismo de la especie. Por otro lado, tomando en cuenta su importancia como especie de consumo y de cultivo, se estaría generando información adicional en cuanto a la contribución de la especie, en el aporte de estos minerales, considerados como esenciales en la alimentación del hombre (13); así como para mejorar las 


\section{MATERIALES Y MÉTODOS}

9 Citavi ha encontrado 1 títulos que se pueden importar directamente. Haga clic aquí para importar los títulos.

capturados en las lagunas Castillero y la Teja, de la región de Caicara del Orinoco, estado Bolívar (FIG. 1). Estos muestreos se realizaron entre los meses de noviembre 1998 y marzo 1999 o meses de sequía, utilizando chinchorros de enmalle de $10 \mathrm{~cm}$ de luz de malla. A cada uno de los peces capturados se les determinó la concentración de potasio, sodio, calcio, magnesio y hierro, y luego un promedio en la concentración de cada uno de ellos.

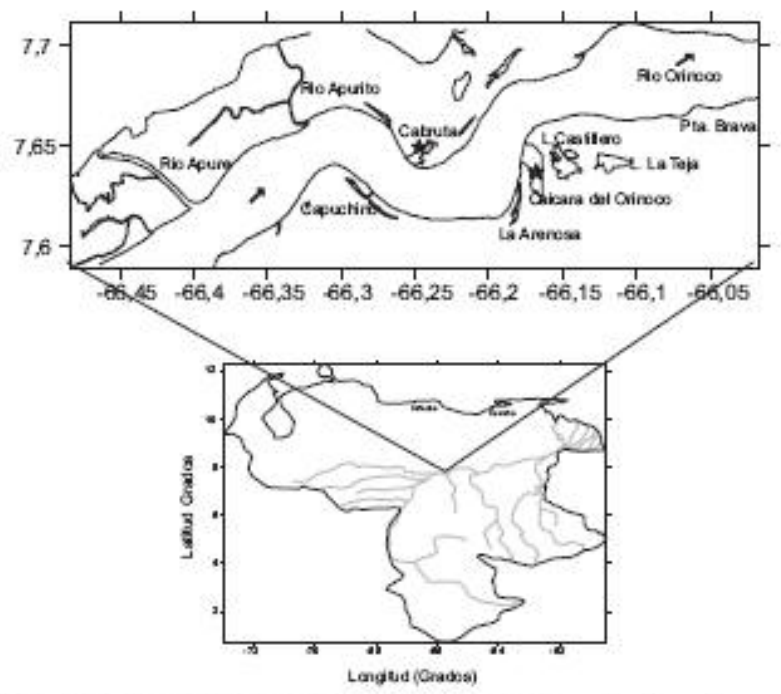

FIGURA 1. ZONAS DE MUESTREO DE Piaractus brachypomUS EN EL ORINOCO MEDIO DE VENEZUELA / AREAS OF SAMPLING OF Piaractus brachypomus IN THE MIDDLE ORINOCO IN VENEZUELA.

Los peces capturados fueron conservados en hielo y en cavas de fibra de vidrio hasta ser trasladados al laboratorio, donde a cada ejemplar se le extrajo una muestra del tejido muscular, a través de cortes realizados por encima de la línea lateral y a nivel del inicio de la aleta dorsal. Los cortes fueron congelados a $-15^{\circ} \mathrm{C}$ en un refrigerador hasta el momento del análisis. Posteriormente fueron secados, a peso constante, a $80^{\circ} \mathrm{C}$ en cápsulas de Petri y en una estufa marca Memmert (Alemania). Los tejidos secos fueron macerados y homogenizados en un mortero de porcelana (Marca, Coors 62012; EUA). Por triplicado, dos gramos de muestra seca fueron pesados en una balanza analítica marca Denver Instrument M-10 EUA, con precisión de 0,0001g; digestadas bajo campana de extracción con ácido nítrico concentrado de calidad analítica ultra pura y posteriormente, enrasada a $25 \mathrm{ml}$ en balones aforados (25).

La cuantificación de los minerales se realizó por Absorción Atómica con llama de aire-acetileno $(11,18,25)$ y corrección de fondo de deuterio, utilizando un equipo Perkin Elmer 3100 (EUA), acoplado con un automuestreador Perkin-Elmer AS-51(EUA). Tanto los patrones de calibración (construidos a partir de ampollas estándares certificadas, marca FIXANAL de fabricación alemana) y los blancos de trabajo, recibieron el mismo tratamiento. Todo el material volumétrico, utilizado en el laboratorio, fue del tipo Pirex de Clase A (EUA).

El agua utilizada, tanto en la preparación de reactivos, curvas de calibración y blancos de reactivos, fue agua desionizada altamente pura (agua calidad NANOPURE de conductividad de $18 \mathrm{MW} / \mathrm{cm}$ ), obtenida con un sistema 
NANOPURE UV, Marca Barnstead (EUA). El equipo Perkin Elmer 3100 (EUA) acoplado con el automuestreador Perkin-Elmer AS-51, exige el uso de agua ionizada; de mayor calidad y precisión en las determinaciones.

9 Citavi ha encontrado 1 títulos que se pueden importar directamente. Haga clic aquí para importar los títulos.

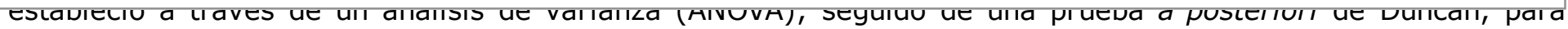
determinar el orden de importancia de dichas concentraciones. La relación de la concentración de los minerales con el peso de los peces, se hizo a través de un análisis de correlación. Estas pruebas estadísticas fueron aplicadas utilizando el programa Statgraphics Plus, versión 4,1. (7).

\section{RESULTADOS Y DISCUSIÓN}

La composición química de los peces puede variar considerablemente entre las diferentes especies y entre los individuos de una misma especie, dependiendo de la edad, sexo, medio ambiente y la época del año (23). Las concentraciones de los minerales determinados en el tejido muscular de $P$. brachypomus, correspondieron a la época de sequía, sin abarcar la época de lluvia por insuficiencia de datos. Durante los meses de lluvia e inundaciones, fue difícil obtener una muestra representativa de la especie debido a que en esa época, tal como ocurre con la mayoría de las especies del Orinoco, disminuyó su accesibilidad hacia el arte de pesca utilizado en los muestreos; de tal manera que se hizo imposible establecer variaciones en la concentración de los minerales, con la época del año. Por la misma razón de insuficiencia de datos, el sexo tampoco fue considerado en el estudio, analizándose de manera individual un total de 40 peces de ambos sexos.

El promedio de las concentraciones de potasio, sodio, calcio, magnesio y hierro están representadas en la TABLA I. Estas concentraciones presentaron variaciones significativas $(P=0,0000 ; a=0,05)$, con una mayor concentración del potasio, seguido del sodio y el calcio y luego, el magnesio y el hierro. Este orden de importancia en la concentración de estos minerales, aparentemente es común para la mayoría de los peces (19); demostrándose en algunas especies como Tilapia nilotica, Cirrhina mrigala y Clarius batrachus, en el lago Dhanmondi de Bangladesh en la India (4). La concentración de potasio fue de $16488,9 \mu \mathrm{g} / \mathrm{g}$, mayor al promedio reportado para los peces en general, el cual fue de $2780 \mu \mathrm{g} / \mathrm{g}$ (19). La concentración de sodio y calcio fue de respectivamente, 3529,66 y 3497,48 $\mathrm{mg} / \mathrm{g}$; aproximadamente iguales a las reportadas para las especies $T$. nilotica, C. mrigala y C. batrachus (4), las cuales presentaron concentraciones de $3183 \mu \mathrm{g} / \mathrm{g}$ de sodio y 4999 $\mu \mathrm{g} / \mathrm{g}$ de calcio. Además de resultar ser los minerales más abundantes, en el tejido muscular de $P$. brachypomus se observó una correlación entre las concentraciones de calcio y potasio $(P=0,0114 ; a=0,05)$, así como también, posiblemente, la concentración de sodio, tomando en cuenta el valor de $\mathrm{P}$ de la prueba estadística (TABLA II). Esta misma correlación se ha demostrado en algunas especies como Oreochromis mossambicus, donde los iones de sodio, potasio y calcio, presentaron mecanismos de transportes semejantes a nivel celular (5). Las concentraciones de los minerales sodio, potasio y calcio en algunos peces, pueden igualmente aumentar cuando se incrementan sus concentraciones en el medio ambiente, provocando, además, un aumento en el consumo de otros elementos como mercurio inorgánico $(14)$, zinc y cadmio $(3,9)$. También pueden influir en el crecimiento y los mecanismos de osmorregulación en los peces (15) y causar hipertrofia de las células branquiales (24).

TABLA I. ANÁLISIS DE RANGO MÚLTIPLE (DUNCAN) DE LAS CONCENTRACIONES DE MINERALES ( $\mu$ g/g) EN EL TEJIDO MUSCULAR DE Piaractus brachypomus $(\mathrm{F}=79878,95 ; \mathrm{P}=0,0000 ; \mathrm{a}=0,05) /$ ANALYSIS OF MULTIPLE RANGE (DUNCAN) OF THE CONCENTRATIONS OF MINERALS ( $\mu \mathrm{g} / \mathrm{g}$ ) IN 
THE MUSCULAR TISSUE OF Piaractus brachypomus $(\mathrm{F}=79878,95 ; \mathrm{P}=0,0000 ; \mathrm{a}=0,05)$

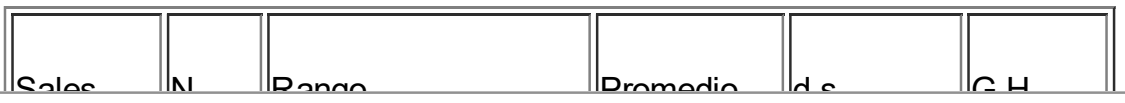

9 Citavi ha encontrado 1 títulos que se pueden importar directamente. Haga clic aquí para importar los títulos.

\begin{tabular}{||l||l||l|l|l||l||}
\hline \hline Hierro & 40 & $43,12-45,99$ & 45,32 & 0,52 & $X$ \\
\hline \hline Magnesio & 40 & $1487,56-1589,65$ & 1528,95 & 29,26 & $X$ \\
\hline \hline Calcio & 40 & $3200,72-3587,00$ & 3497,48 & 107,062 & $X$ \\
\hline \hline Sodio & 40 & $3203,43-3591,23$ & 3529,66 & 65,24 & $X$ \\
\hline \hline Potasio & 40 & $15895,30-16984,30$ & 16488,90 & 264,69 & $X$ \\
\hline \hline
\end{tabular}

$\mathrm{N}$ = Tamaño de la muestra. d.s = Desviación estándar. $\mathrm{G} \cdot \mathrm{H}=$ Grupos homogéneos

TABLA II. CORRELACIÓN $(a=0,05)$ ENTRE LAS CONCENTRACIONES DE MINERALES Y EL PESO DE Piaractus brachypomus / CORRELATION $(a=0,05)$ AMONG THE CONCENTRATIONS OF MINERALS AND THE WEIGHT OF Piaractus brachypomus

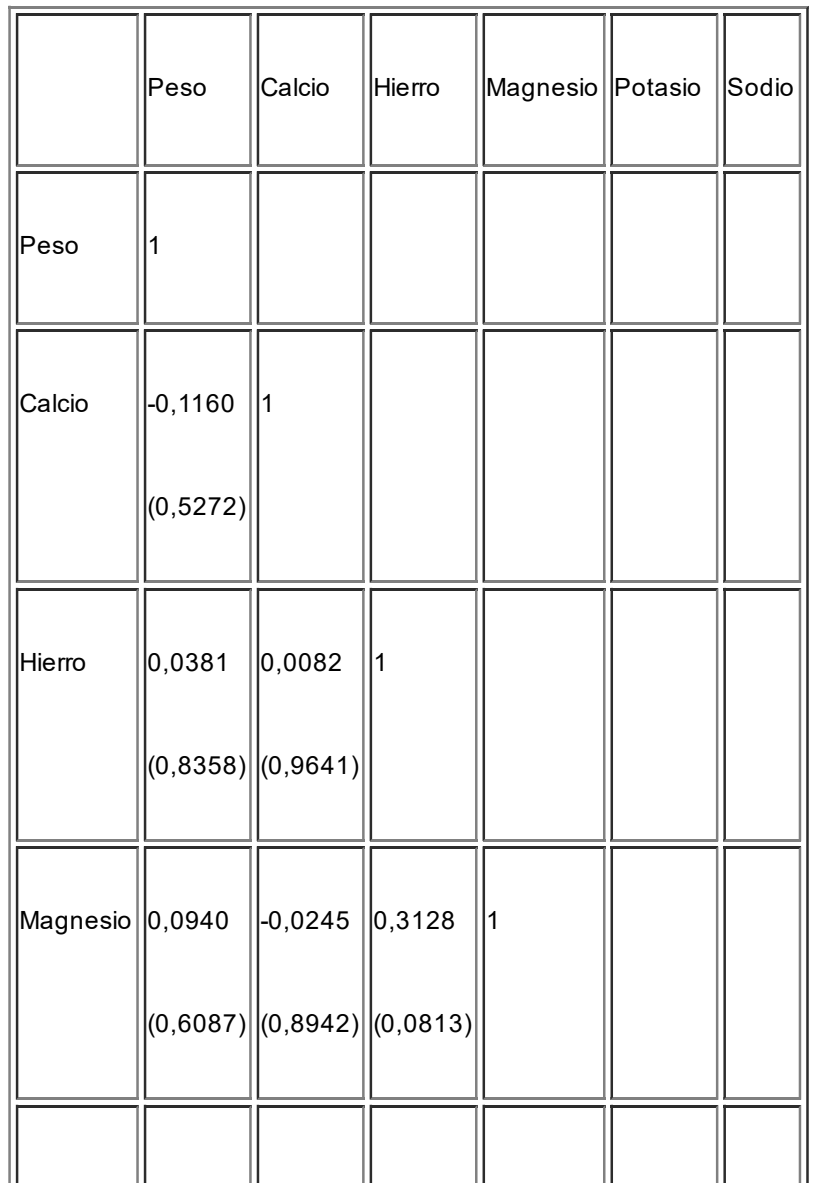




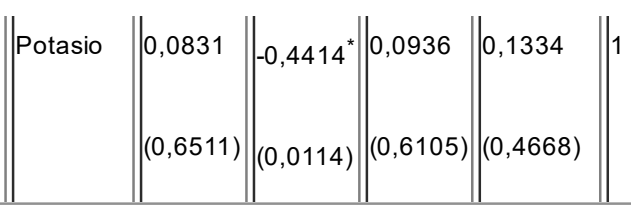

Citavi ha encontrado 1 títulos que se pueden importar directamente. Haga clic aquí para importar los títulos.

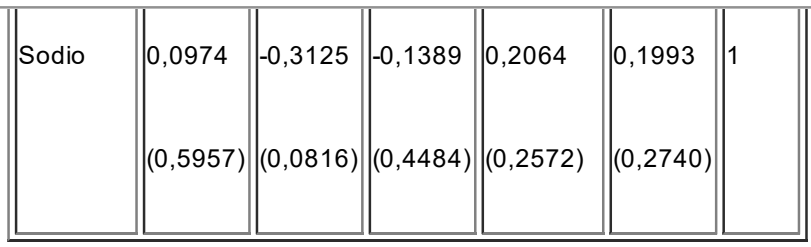

Correlación. (Valor de P). * Significativamente correlacionadas.

Aunque no se observó significativamente en el trabajo, en algunas especies, el calcio, el sodio y el potasio, también guardan cierta relación con el magnesio, ya que un aumento en la concentración de este último puede incidir en la homeostasis de los primeros (8). La concentración de magnesio en el tejido muscular de $P$. brachypomus fue de 1528,95 $\mu \mathrm{g} / \mathrm{g}$, aproximadamente igual a la concentración reportada para las especies $T$. nilotica, C. mrigala y C. batrachus, la cual fue de $2193 \mu \mathrm{g} / \mathrm{g}$ (4). Este mineral, es importante en el funcionamiento de las enzimas que intervienen en el desdoblamiento de la glucosa (8), mientras que un aumento en su concentración, por incremento de la concentración en el ambiente, puede interferir en la absorción y acumulación de otros minerales como el zinc (1).

El mineral que presentó menor concentración en el tejido muscular de $P$. brachypomus fue el hierro (45,32 $\mu \mathrm{g} / \mathrm{g}$ ), contradiciendo las altas concentraciones que presenta este mineral en las aguas superficiales $y$ sedimento del Orinoco medio (10). Lo mismo se ha demostrado para otras especies como Perca fluviatilis y Rutilus rutilus, en las cuales la concentración de hierro fue baja, a pesar de vivir en ambientes de altas concentraciones (24). Algunas condiciones de acidez y oxígeno, facilitan la asimilación del hierro por parte de estas especies, en medios que presentan altas concentraciones (2). La concentración de hierro en el tejido muscular de $P$. brachypomus fue incluso menor que la reportada para otras especies como $T$. nilotica, $C$. mrigala y C. batrachus, en las cuales la concentración alcanzó un promedio de 131 $\mathrm{g} / \mathrm{g}$ (4).

Tal como se señaló anteriormente, las concentraciones de minerales en los peces varían con la edad (23), sin embargo, en $P$. brachypomus no se observó correlación alguna entre las concentraciones de los minerales y el peso de los ejemplares (TABLA II). Posiblemente el pequeño rango de tallas considerado en el trabajo, pudo haber influido en este resultado, recomendándose analizar mayores categorías de tallas, para establecer exactamente una posible variación en la concentración de estos minerales, con el tamaño de los peces.

Como información adicional desde el punto de vista nutritivo, $P$. brachypomus contiene relativamente altas concentraciones de potasio, sodio, calcio y magnesio, minerales considerados como esenciales en la alimentación del ser humano (13) y presentes en algunos alimentos ingeridos por el hombre (6); de allí que su consumo constituya un aporte de estos minerales en la nutrición del venezolano, además de la exquisitez de su carne. Por otro lado, los resultados también podrían ser utilizados como información en la acuacultura, para diseñar el alimento y mejorar la calidad de la carne. La composición química de los peces cultivados puede predecirse, cuando se seleccionan ciertas condiciones de cultivo como la composición del alimento, además de 
las condiciones ambientales, el tamaño de los peces y los rasgos genéticos; los cuales tienen impacto en la composición y calidad de los peces cultivados (12).

9 Citavi ha encontrado 1 títulos que se pueden importar directamente. Haga clic aquí para importar los títulos.

En el tejido muscular de $P$. brachypomus el mineral más importante fue el potasio, seguido del sodio y el calcio, el magnesio y el hierro; los cuales son importantes en el metabolismo de la especie en concentraciones normales. Cuando aumenta su concentración por incremento de la condensación en el medio ambiente, se puede alterar el metabolismo y estimular el consumo de otros minerales que pueden resultar tóxicos para los peces e incluso para el ser humano.

Generalmente las concentraciones de minerales como potasio, sodio, calcio, magnesio y hierro aumentan con el tamaño de los peces, sin embargo en el presente trabajo dichas concentraciones no presentaron correlación alguna con el tamaño de estos peces, aunque el pequeño rango de tallas considerado pudo incidir en los resultados. Se recomienda ampliar las categorías de tallas, para verificar si en realidad existe o no una correlación entre el contenido de estos minerales con el tamaño de los peces.

También se debería aumentar el tamaño de la muestra para estratificar el estudio por sexo y abarcar, en lo posible, la época de lluvia e inundaciones; así se podrían establecer posibles variaciones en la concentración de estos minerales con el sexo y con la época de sequía e invierno de la región del Orinoco medio.

La concentración de los minerales analizados en $P$. brachypomus caen dentro del rango de concentración señalado para los peces de agua dulce, e incluso, la concentración de algunos minerales como el potasio fue muy superior. De allí que tomando en cuenta que estos minerales son considerados como esenciales para los seres vivos y que forman parte importante de algunos alimentos ingeridos por el hombre, el consumo de $P$. brachypomus, además de la exquisitez de su carne, constituye un aporte de dichos minerales en la alimentación del venezolano.

\section{AGRADECIMIENTO}

A Fundacite-Guayana, por el financiamiento del proyecto que dio origen a la presente investigación, así como al Instituto Limnológico de la Universidad de Oriente, por la logística prestada.

\section{REFERENCIAS BIBLIOGRÁFICAS}

1. ADHIKARI, S. Interference of magnesium on zinc adsorption by pond sediment and on zinc accumulation in a freshwater teleost, Labeo rohita (Hamilton). Ecotoxicol. and Environment Safety. 59 ( 2): 228-231. 2004. [ L Links ]

2. ANDERSEN, O. Accumulation of waterborn iron and expresión of firritin and transferrin in early developmental stages of brown trout Salmo trutta. Fish Physiol. and Biochem. 16 ( 3): 223-231. 1997.

3. BALDISSEROTTO, B.; CHOWDHURY, M.J.; WOOD, C.M. Effects of dietary calcium and cadmium on cadmium accumulation, calcium and cadmium uptake from the water, and their interactions in juvenile rainbow trout. Aquatic. Toxicol. 72 ( 1-2): 99-117. 2005. 
4. BEGUM, A.; AMIN, N.; KANEKO, S.; OHTA, K. Selected elemental composition of the muscle tissue of three species of fish, Tilapia nilotica, Cirrhina mrigala and Clarius batrachus, from the fresh water Dhanmondi Lake in Bangladesh. Food Chem. 93 ( 3): 439-443. 2005.

[ Links ]

9 Citavi ha encontrado 1 títulos que se pueden importar directamente. Haga clic aquí para importar los títulos.

5. BIJVELUS, M.J.C.; FLIK, G.; VVEIVUELAAR, S.E. IMIneral Dalance in Ureocnromis mossamDICUS: aepenaence on magnesium in diet and water. Fish Physiol. and Biochem. 16 (4): 323-331. 1997.

6. DASHTI, B.; AL-AWADI, F.; ALKANDARI, R.; ALI, A.; AL-OTAIBI, J. Macro-and microelements contents of 32 Kuwaiti composite dishes. Food Chem. 85 (3): 331-337. 2004.

[ Links ]

7. FERNÁNDEZ, P.F.; LÓPEZ, S.M.A.; MUÑOZ, M.M.; RODRÍGUEZ, C.A.M.; SÁNCHEZ, N.A.; VALERO, F.C. Libro: Estadística asistida por ordenador Statgraphics Plus 4,1. Universidad de Cadiz, Servicio de Publicaciones, España. En línea: http: //www2.uca.es/grup-invest/teloydisren/alumnus/eapo/eapo.htm-2k. 2004. [ [ Links ]

8. FLIK, G.; KANEKO, T.; GRECO, A.M.; LI, J.; FENWICK, J.C. Sodium dependent ion transporters in trout gills. Fish Physiol. and Biochem. 17 ( 1-6): 385-396. 1997. [ Links ]

9. GLOVER, C.N.; BURY, N.R.; HOGSTRAND, C. Intestinal Zinc uptake in freshwater rainbow trout: evidence for apical pathways associated with efflux and modified by calcium. Bioch. et Biophy. Acta (BBA)-Biomembr. 1663 ( 1-2): 214-221. 2004.

[ Links ]

10. GONZÁleZ, A.; MÁRQUeZ, A.; CHUNG, K.S. Hierro y cobre en Plagioscion squamosissimus (Pises: Sciaenidae) del río Orinoco, Venezuela. Rev. Biol. Trop. 48 ( 1): 207-213. 2000. [ Links ]

11. HANSEN, C.; NIELSEN, C.; DIETZ, R; HANSEN, M. Zinc, cadmium, mercury and selenium in minke whales, belugas and narwhals from west Greenland. Polar Biol. 10: 529-539. 1990.

[ Links ]

12. HUSS, H. H. Quality and quality changes in freshwater fish. FAO Fish. Techn. Paper No. 348.203 pp. 1995. [ L Links ]

13. ITRIAGO, A.; CARRIÓN, N.; FERNÁNDEZ, A.; PUIG, M.; DiNÍ, E. Contenido de zinc, cobre, calcio, fósforo y magnesio en la leche materna en los primeros días de lactación. Arch.Latinoam. Nutri. 47 ( 1): 14-22. 1997. [ L Links ]

14. KLINCK, J.; DUNBAR, M.; BROWN, S.; NICHOLS, J.; WINTER, A.; HUGHES, C.; PLAYLE, R.C. Influence of water chemistry and natural organic matter on active and passive uptake of inorganic mercury by gills of rainbow trout (Oncorhynchus mykiss). Aquatic Toxicol. 72 ( 1-2): 161-175. 2005.

[ Links ]

15. LAIZ-CARRION, R.; SANGIAO-ALVARELlOS, S.; GUZMAN, J.M.; MARTÍN DEL RÍO, M.P.; SOENGAS, J.L.; MANCERA, J.M. Growth performance of gilthead sea bream Sparus aurata in different osmotic conditions: Implications for osmoregulation and energy metabolism. Aquacult. 250 ( 3-4): 849-861. 2005.

[ Links ]

16. LOVSHIN, L.L. The colossomids. In: Nash, C.E.; Novotny, A.J. (Eds). World animal science: Production of aquatic animals: Fishes. Elsevier Science, Amsterdam, The Netherlands: 153-159 pp. 
1995. [ Links ]

17. MACHADO-ALLISON, A. Los peces de los llanos de Venezuela. Un ensayo sobre su Historia Natural.

9 Citavi ha encontrado 1 títulos que se pueden importar directamente. Haga clic aquí para importar los títulos.

1993. [ LINKS ]

18. MALCOM, H.; BOYD, I.; OSBORN, D.; FRENCH, M.; Freestone, P. Trace metal in Antartic fur seal (Artocephalus gazella) livers from Bird Island, South Georgia. Mar. Pollu. Bull. 28: 375-380. 1994.

[ Links ]

19. MURRAY, J.; BURT, J.R. Vitamins and minerals in fish. Food and Agriculture Organization of the United Nations (FAO). Organization of the United Nations. Roma. En línea: www.fao.org/DOCREP/V7180S/v7180s0.5.htm. $1998 . \quad$ [ Links ]

20. NOVOA, D. Análisis histórico de las pesquerías del Río Orinoco. En: Novoa, D. (Ed). Los recursos pesqueros del Río Orinoco y su explotación. Caracas. CVG. 1-386pp. 1982. [ [ Links ]

21. NOVOA, D.; RAMOS, F.; CARTAYA, E. Las pesquerías artesanales del Río Orinoco, Sector Caicara- Cabruta. Parte I. Mem. Soc. Cienc. Nat. La Salle. 121 (44): 163-215. 1984.

[ Links ]

22. NOVOA, R.D.F. Los recursos pesqueros del eje fluvial Orinoco-Apure: Presente y Futuro. Ministerio de Agricultura y Tierra, INAPESCA, Venezuela. 1-148 pp. 2002.

[ Links ]

23. STANSBY, M.E. Composición química de los peces. Food and Agriculture Organization of the United Nations (FAO). Organization of the United Nations. Roma. En línea: www.fao.org/DOCREP/V7180S/v7180s0.5.htm. 1998.

[ Links ]

24. TKATCHEVA, V.; HYVÄRINEN, H.; KUKKONEN, J.; RYZHKOV, L.P.; HOLOPAINEN, I.J. Toxic effects of mining effluents on gills in a subarctic lake system in NW Russia. Ecotoxicol. and Envirom. Safety. 57 ( 1 ): 1-8. 2004. [ Links ]

25. WOOD, C.; VAN VLEET, E. Cooper, cadmium and zinc in the liver, kidney and muscle tissues of bottlenose dolphins (Tursiops truncatus) stranded in Florida. Mar. Poll. Bull. 32 ( 12): 886-889. 1996.

[ Links ]

Facultad de Ciencias Veterinarias, Universidad del Zulia, Núcleo Agropecuaria Ciudad Universitaria. Apdo. 15252, Maracaibo 4005-A. Estado Zulia-Venezuela. Telf. Fax: 58261-7596158.

efMail

revistafcvluz@hotmail.com 